\title{
A Comparative Study of Treatment Toxicities Between FOLFOX 4 and Modified FOLFOX 6 in Iranian Colorectal Cancer Patients
}

\author{
Amir Shahram Yousefi Kashi, ${ }^{1}$ Abolfazl Razzaghdoust, ${ }^{2}$ and Afshin Rakhsha ${ }^{1,{ }^{*}}$ \\ ${ }^{1}$ Department of Radiation Oncology, Shohada-e-Tajrish Hospital, Faculty of Medicine, Shahid Beheshti University of Medical Sciences, Tehran, IR Iran \\ ${ }^{2}$ Cancer Researcher, Department of Radiation Oncology, Shohada-e-Tajrish Hospital, Shahid Beheshti University of Medical Sciences, Tehran, IR Iran \\ "Corresponding author: Afshin Rakhsha, Department of Radiation Oncology, Shohada-e-Tajrish Hospital, Faculty of Medicine, Shahid Beheshti University of Medical Sciences, \\ Tehran, IR Iran. Tel/Fax: +98-2122739200, E-mail: afshinrakhsha@gmail.com
}

Received 2016 October 21; Revised 2016 November 06; Accepted 2017 January 03.

\begin{abstract}
Background: Colorectal cancer is one major health problem and cancer-related cause of death in cancer patients in countries such as Iran where the most cases are diagnosed in advanced stages.

Objectives: To evaluate the incidence and severity of toxic effects in colorectal cancer patients who have been treated with two different schedules of combination of oxaliplatin and bolus/infusional 5-fluorouracil with leucovorin (FOLFOX) and to compare them.

Methods: Medical records of 458 patients with colorectal cancer treated with FOLFOX 4 and modified FOLFOX 6 regimen between 2005 and 2014 were reviewed. Data from 96 eligible patients were analyzed. Fifty-six patients (58.3\%) received FOLFOX 4 and 40 patients (41.7\%) received modified FOLFOX 6.

Results: The study included 96 patients, 39 of whom were males (40.6\%) and 57 of whom were females (59.4\%). The median age was 62 years (range: 38 - 87 years). The follow up duration was between 16 - 109 months with a median of 62 months. There was a statistically significant incidence rate of grade $\geq 1$ toxicity of diarrhea as gastrointestinal (GI) toxicity between FOLFOX 4 and modified FOLFOX 6 as the two regimens $(P=0.034)$, but there was not a statistically significant incidence rate of grade $\geq 1$ toxicity of stomatitis as GI toxicity between the two regimens $(\mathrm{P}=0.27)$. We observed a highly statistically significant incidence rate of grade $\geq 1$ toxicity of neutropenia as hematologic toxicity between FOLFOX 4 and modified FOLFOX 6 as the two regimens $(\mathrm{P}<0.001)$, but we did not observe any statistically significant differences of grade $\geq 1$ of thrombocytopenia as hematologic toxicity between the two regimens $(P=0.063)$. There was a statistically significant incidence rate of grade $\geq 1$ neurotoxicity between FOLFOX 4 and modified FOLFOX 6 as the two regimens $(\mathrm{P}=0.017)$.

Conclusions: We showed that in colorectal cancer patients treated with modified FOLFOX6. Some of hematological and nonhematological complications were more than FOLFOX4 and they can be concerned.
\end{abstract}

Keywords: FOLFOX4, Modified FOLFOX 6, Toxicities, Colorectal Cancer

\section{Background}

Colorectal cancer (CRC) is the third diagnosed solid cancer in the world and Iran (1). Surgery, radiotherapy and chemotherapy are the main therapeutic interventions for CRC. Among the chemotherapy regimens, combination of oxaliplatin is a platinum analogue and bolus/infusional 5fluorouracil (5-FU) with leucovorin (LV), called FOLFOX, is the most-studied and effective chemotherapeutic regimen in adjuvant and metastatic settings.

FOLFOX has effectively increased the progression free survival (PFS) in colorectal cancer patients (2). There are two common types of FOLFOX regimens: FOLOFOX 4 and modified FOLFOX 6 (mFOLFOX6) (3).

Based on the results of the MOSAIC studies, FOLFOX4 is known as the best standard of care in adjuvant chemotherapy for Stage III colorectal cancer in Western countries (4). Since then, the FOLFOX regimens have been used in differ- ent randomized clinical trials with bevacizumab or cetuximab or panitumumab. They have been shown to the establishment of other schedules of FOLFOX regimens (5-7).

However, mFOLFOX6 that can be infused easily by a venous catheter or port that can be avoided of long hospitalization, and is known as equally effective as FOLFOX 4 by the oncologic centers (8).

There are a few studies that reported the incidence rate of severity of adverse effects such as neutropenia, thrombocytopenia, neurotoxicity and diarrhea induced by chemotherapy in colorectal cancer patients (9).

With the addition of oxaliplatin to $5 \mathrm{FU} / \mathrm{LV}$ chemotherapy, the incidence rate of hematological and nonhematological complications is increased significantly (10). FOLFOX4 is associated with grade 3 or 4 diarrhea and stomatitis that can be well controlled with effective supportive protocol (11). 


\section{Objectives}

In the current study, we evaluated and compared the incidence and severity of chemotherapy induced neutropenia, thrombocytopenia, stomatitis, diarrhea and neurotoxicity adverse effects in patients treated with FOLFOX 4 and modified FOLFOX 6.

\section{Methods}

This was a cross sectional-analytic study where data obtained from medical records of 458 patients with colorectal cancer treated with FOLFOX 4/6 regimen between 2006 and 2014 were reviewed.

A total of 96 eligible patients diagnosed with histopathologically confirmed carcinoma of colorectal at the radiation-oncology ward of Shohada-e-Tajrish hospital between February 2005 and March 2014 were included and followed.

The inclusion criteria were patients with histopathology diagnosis of colorectal cancer with or without distant metastasis who were treated by chemotherapy at our center, adequate white blood count and platelet before therapy, ages 18 - 80 years, estern cooperative oncology group(ECOG) performance score 0 -2 and acceptable liver and renal function tests. Finally, ninety-six patients were selected in the study as per defined criteria.

Patients were excluded from the study if they were without a histopathology diagnosis other than colorectal cancer, if they had undergone neo-adjuvant or adjuvant treatments such as radiation therapy or chemotherapy initiated by another radiation oncology center, and if they were without follow-up.

The first cycle of FOLFOX chemotherapy was administered based on our inclusion criteria described above. Subsequent cycles were not allowed to infusion unless white blood cells were $\geq 1,500 / \mathrm{mm}^{3}$, platelet count $\geq$ $100,000 / \mathrm{mm}^{3}$ and non-hematological toxicities recovered to grade zero or one.

All adverse effects were recorded according to national cancer institute common toxicity criteria (NCI CTC) version 2.0 grading scale on a scale of 1 to 5 according to the general grade definition. The defined parameters of hematological and non-hematological complications in our study were neutropenia, thrombocytopenia, neurotoxicity, diarrhea and stomatitis that were clinically evaluated after each treatment cycle of chemotherapy.

Two different regimens of oxaliplatin with 5FU/LV(FOLFOX) were administered as chemotherapy protocols: FOLFOX4 $(\mathrm{n}=56)$ : Oxaliplatin: $85 \mathrm{mg} / \mathrm{m}^{2}$ intra venus (IV) on the first day in 500 cc of dextrose water $5 \%$ in 2 hours via a Y connector concurrently with LV as rescue factor: 200 $\mathrm{mg} / \mathrm{m}^{2} \mathrm{IV}$ in 2 hours infusion before 5FU bolus and 5FU: 400 $\mathrm{mg} / \mathrm{m}^{2}$ IV bolus, followed by $600 \mathrm{mg} / \mathrm{m}^{2}$ IV continuous infusion for 22 hours on the first and second days. (Cycle repeated every two weeks up to 12 cycles). MFOLFOX6 ( $\mathrm{n}=$ 40): Oxaliplatin: $85 \mathrm{mg} / \mathrm{m}^{2}$ IV on the first day in $500 \mathrm{cc}$ of dextrose water $5 \%$ in 2 hours via a Y connector plus LV 400 $\mathrm{mg} / \mathrm{m}^{2}$ IV over 2 hours on day 1 and 5FU bolus: $400 \mathrm{mg} / \mathrm{m}^{2}$ IV followed by $5 \mathrm{FU}$ infusional: $2400 \mathrm{mg} / \mathrm{m}^{2}$ IV continuous infusion for 46 hours on the first and second days. (Cycle repeated every two weeks up to 12 cycles).

The ethical regulations dictated in the act provided by Shahid Beheshti University of Medical Sciences (reference number of research ethics committee: 429) were strictly observed. The data were strictly preserved without using the patients' names.

Pearson's $\chi^{2}$ test was used to compare the number of patients with toxicities between two regimens. Fisher's exact test was used to adjust $\chi^{2}$ probability values when cell frequencies were lower than the expected values. The statistical analyses were performed by using software SPSS version 21 (SPSS IBM).

\section{Results}

The study included 96 patients, 39 of whom were males (40.6\%) and 57 of whom were females (59.4\%). The median age was 62 years (range: 38 - 87 years). The follow up duration was between 16 - 109 months with a median of 62 months. Fifty-six patients $(58.3 \%)$ received FOLFOX 4 and 40 patients (41.7\%) received modified FOLFOX 6. In FOLFOX 4 group, 29 patients (55\%) had colon cancer and in modified FOLFOX 6 group, 23 patients (45\%) had colon cancer. In FOLFOX 4 group, 27 patients (61.4\%) had rectal cancer and in modified FOLFOX 6 group, 17 patients (38.6\%) had rectal cancer.

Pathological T stages were 54.5\% T2 in FOLFOX 4 and $45.5 \%$ in modified FOLFOX 6, 58.4\% T3 in FOLFOX 4 and $41.6 \%$ in modified FOLFOX 6 and 62.5\% T4 in FOLFOX 4 and 37.5\% in modified FOLFOX 6.

Pathological N stages were negative in $62.9 \%$ of FOLFOX 4 patients and 37.1\% in modified FOLFOX 6 patients; Pathological N stages were positive in $55.3 \%$ of FOLFOX 4 patients and $44.7 \%$ in modified FOLFOX 6 patients. Pathological $\mathrm{N}$ stages were unknown in $57.1 \%$ of FOLFOX 4 patients and $42.9 \%$ in modified FOLFOX 6 patients. Clinical M stages were negative in $50.9 \%$ of FOLFOX 4 patients and $49.1 \%$ in modified FOLFOX 6 patients and were positive in $58.3 \%$ of FOLFOX 4 patients and $41.7 \%$ in FOLFOX 6 patients. Clinical M stages were unknown in $74.1 \%$ of FOLFOX 4 patients and $25.9 \%$ in modified FOLFOX 6 patients.

The total number of chemotherapy cycles in FOLFOX 4 and mFOLFOX6 groups were 516 and 384 respectively. The 
median number of chemotherapy cycles in FOLFOX 4 and mFOLFOX6 groups were 10 and 8 respectively. 11 (55\%) patients had received 4 - 7 cycles of FOLFOX 4 and 9 (45\%) patients had received 4-7 cycles of modified FOLFOX 6. 45 (59.2\%) patients had received 8 - 12 cycles of FOLFOX 4 and thirty-one $(40.8 \%)$ patients had received $8-12$ cycles of modified FOLFOX 6.

Treatment delay was observed in 6 patients $(28.6 \%)$ of FOLFOX 4 group and in 15 patients (71.4\%) of modified FOLFOX 6 group. There was no need to dose adjustment in FOLFOX 4 group and dose adjustment was needed in 5 patients in FOLFOX 6 group. There was no need to stop our treatment in FOLFOX 4 group and mFOLFOX 6 group permanently. The patient characteristics and treatment-related parameters are shown in Table 1.

The most complicating effects reported in FOLFOX 4 group were diarrhea (19.6\%) and neurotoxicity (19.6\%) of grade 1 and more. Grade $\geq 1$ of neutropenia and thrombocytopenia were reported in $8.9 \%$ and $25 \%$ cases respectively. Grade $\geq 1$ of stomatitis was reported in $8.9 \%$ patients. The incidence rate and complication grade of the adverse effects with relative frequency rate are shown in Figure 1.



Figure 1. Distribution of Different Toxicities Between the Two Regimens \% of Patients With $\geq$ Grade 1 Toxicity

The most frequent adverse effect reported in the patients of modified FOLFOX 6 was neutropenia (80\%) of grade 1 and more. Grade 1 and more of thrombocytopenia were reported in 55\% of modified FOLFOX6 arm. Grade $\geq 1$ of neurotoxicity was reported in $75 \%$ of modified FOLFOX6 arm. Grade $\geq 1$ of stomatitis and diarrhea were reported in $32.5 \%$ and $75 \%$ cases respectively. The incidence rate and the toxicity grades of the symptoms with relative frequency are shown in Figure 1.

There was a statistically significant incidence rate of grade $\geq 1$ toxicity of diarrhea as gastrointestinal (GI) toxicity between FOLFOX 4 and modified FOLFOX 6 as the two regimens $(\mathrm{P}=0.034, \mathrm{OR}=3.16,95 \% \mathrm{CI}=1.05-9.44)$, but there was not a statistically significant incidence rate of grade $\geq$ 1 toxicity of stomatitis as GI toxicity between the two regimens $(\mathrm{P}=0.27, \mathrm{OR}=2.52,95 \% \mathrm{CI}=0.56-11.23)$. Then, FOLFOX 4 scheme was statistically significant better than modified FOLFOX 6 scheme of diarrhea as GI toxicity.

We observed a very highly statistically significant incidence rate of grade $\geq 1$ toxicity of neutropenia as hematologic toxicity between FOLFOX 4 and modified FOLFOX 6 as the two regimens $(\mathrm{P}<0.001, \mathrm{OR}=8.50,95 \% \mathrm{CI}=2.23$ 32.42), but we did not observe any statistically significant differences of grade $\geq 1$ of thrombocytopenia as hematologic toxicity between two regimens $(\mathrm{P}=0.063, \mathrm{OR}=2.96$, $95 \% \mathrm{CI}=0.90$ - 9.64). Then, FOLFOX 4 scheme was statistically significant better than modified FOLFOX 6 scheme of neutropenia as hematologic toxicity.

There was a statistically significant incidence rate of grade $\geq 1$ neurotoxicity between FOLFOX 4 and modified FOLFOX 6 as the two regimens $(\mathrm{P}=0.017, \mathrm{OR}=3.57,95 \% \mathrm{CI}=$ 1.20 -10.55). Then, FOLFOX 4 scheme was statistically significant better than modified FOLFOX 6 scheme of neurotoxicity. The difference in the incidence rate of all toxicities between FOLFOX 4 and modified FOLFOX 6 are shown in Table 2.

\section{Discussion}

Hematological and non-hematological toxicities manifested by 5-Fu and oxaliplatin based chemotherapy could be exacerbated by symptoms of neutropenia, thrombocytopenia, diarrhea, stomatitis and neurotoxicity. These toxicities may alter quality of life in cancer patients undergoing chemotherapy (11).

In this study, we compared the differences in hematologic and non-hematologic toxicity rates between FOLFOX 4 and modified FOLFOX 6 in Iranian colorectal cancer. Literature concerning the effectiveness and toxicity of modified FOLFOX regimens compared with FOLFOX 4 regimens (standard of care) is limited. Toxicity can vary based on doses of each chemotherapy drugs and infusion schedules $(12,13)$.

It seems diarrhea and stomatitis are the two most frequent and dose limiting of GI toxicity in colorectal cancer patients $(12,13)$. There was statistically significant difference in grade $\geq 1$ toxicity of diarrhea as GI toxicity, between FOLFOX 4 and modified FOLFOX 6 chemotherapy regimens in our study $(\mathrm{P}=0.034)$. Then, FOLFOX 4 scheme was statistically significant better than modified FOLFOX 6 scheme of diarrhea as GI toxicity. 
Table 1. Patient Characteristics and Treatment-Related Parameters ${ }^{\mathrm{a}}$

\begin{tabular}{|c|c|c|}
\hline & FOLFOX $4(n=56)$ & $\operatorname{mFOLFOX~} 6(n=40)$ \\
\hline \multicolumn{3}{|l|}{ Gender } \\
\hline Female & $35(61.4)$ & $22(38.6)$ \\
\hline Male & $21(53.8)$ & $18(46.2)$ \\
\hline \multicolumn{3}{|l|}{ Cancer type } \\
\hline Colon & $29(55)$ & $23(45)$ \\
\hline Rectum & $27(61.4)$ & $17(38.6)$ \\
\hline \multicolumn{3}{|l|}{ Tumor stage } \\
\hline T II & $6(54.5)$ & $5(45.5)$ \\
\hline T III & $45(58.4)$ & $32(41.6)$ \\
\hline TIV & $5(62.5)$ & $3(37.5)$ \\
\hline \multicolumn{3}{|l|}{ Nodal status } \\
\hline $\mathrm{N}-$ & $22(62.9)$ & $13(37.1)$ \\
\hline $\mathrm{N}+$ & $26(55.3)$ & $21(44.7)$ \\
\hline $\mathrm{N}_{\mathrm{X}}$ & $8(57.1)$ & $6(42.9)$ \\
\hline \multicolumn{3}{|c|}{ Metastasis status } \\
\hline мо & $29(50.95)$ & $28(49.1)$ \\
\hline M1 & $7(58.3)$ & $5(41.7)$ \\
\hline $\mathrm{M}_{\mathrm{X}}$ & $20(74.1)$ & $7(25.9)$ \\
\hline \multicolumn{3}{|c|}{ Previouschemotherapy } \\
\hline Yes & $7(30)$ & $16(70)$ \\
\hline No & $49(67.1)$ & $24(32.9)$ \\
\hline \multicolumn{3}{|c|}{ Courses number } \\
\hline $4-7$ & $11(55)$ & $9(45)$ \\
\hline $8-12$ & $45(59.2)$ & $31(40.8)$ \\
\hline \multicolumn{3}{|c|}{ Treatment delay } \\
\hline Yes & $6(28.6)$ & $15(71.4)$ \\
\hline No & $50(65.3)$ & $25(34.7)$ \\
\hline \multicolumn{3}{|c|}{ Dose adjustment } \\
\hline Yes & 0 & $5(100)$ \\
\hline No & $56(61.5)$ & $35(38.5)$ \\
\hline
\end{tabular}

${ }^{\mathrm{a}}$ Values are expressed as No. (\%).

Diarrhea is usually seen in the third week after the first cycle of chemotherapy with Oxaliplatin with $5 \mathrm{FU}$, which shows that oxaliplatin is the major risk factor of GI toxicities (14). In the case of mFOLFOX 6, there were reports of grade 3 and grade 4 diarrhea or stomatitis. During our study, the most adverse effects induced by chemotherapy were less than grade 2 toxicity, the difference in the incidence rate of grades 3 and 4 toxicities with all grades of toxicity is highly significant also reported earlier $(15,16)$.
There was less adverse effects of diarrhea among FOLFOX4 group in comparison with mFOLFOX 6 group in our study. Diarrhea as therapeutic toxicity highly depends on doses of 5FU infusion administration (17) and we used 600 $\mathrm{mg} / \mathrm{m}^{2} \mathrm{IV}$ continuous infusion for 22 hours on the first and second days in FOLFOX 4 group where less than 1200 $\mathrm{mg} / \mathrm{m}^{2} \mathrm{IV}$ continuous infusion for 22 hours on the first and second days in mFOLFOX 6 group can explain why there was a high incidence rate of diarrhea in the colorectal can- 
Table 2. Distribution of Different Toxicities Between the Two Regimens ${ }^{\mathrm{a}}$

\begin{tabular}{|c|c|c|c|c|}
\hline & \multicolumn{2}{|c|}{ Toxicity } & \multirow[t]{2}{*}{ OR $(95 \% \mathrm{CI})$} & \multirow[t]{2}{*}{ P Value } \\
\hline & Grade 0 & Grade $\geq 1$ & & \\
\hline Neurotoxicity & & & \multirow{4}{*}{$3.57(1.20-10.55)$} & \multirow{4}{*}{0.017} \\
\hline FOLFOX 4 & $50(89.3)$ & $6(10.7)$ & & \\
\hline mFOLFOX 6 & $28(70)$ & $12(30)$ & & \\
\hline Total & $78(81.2)$ & $18(18.8)$ & & \\
\hline Neutropenia & & & \multirow{4}{*}{$8.50(2.23-32.42)$} & \multirow{4}{*}{$<0.001$} \\
\hline FOLFOX 4 & $53(94.6)$ & $3(5.4)$ & & \\
\hline mFOLFOX 6 & $27(67.5)$ & $13(32.5)$ & & \\
\hline Total & $80(83.3)$ & $16(16.7)$ & & \\
\hline Thrombocytopenia & & & \multirow{4}{*}{$2.96(0.90-9.64)$} & \multirow{4}{*}{0.063} \\
\hline FOLFOX 4 & $51(91.1)$ & $5(8.9)$ & & \\
\hline mFOLFOX 6 & $31(77.5)$ & $9(22.5)$ & & \\
\hline Total & $82(85.4)$ & $14(14.6)$ & & \\
\hline Diarrhea & & & \multirow{4}{*}{$3.16(1.05-9.44)$} & \multirow{4}{*}{0.034} \\
\hline FOLFOX 4 & $50(89.3)$ & $6(10.7)$ & & \\
\hline mFOLFOX 6 & $29(72.5)$ & $11(27.5)$ & & \\
\hline Total & $79(82.3)$ & $17(17.7)$ & & \\
\hline Stomatitis & & & \multirow{4}{*}{$2.52(0.56-11.23)$} & \multirow{4}{*}{0.27} \\
\hline FOLFOX 4 & $53(94.6)$ & $3(5.4)$ & & \\
\hline mFOLFOX 6 & $35(87.5)$ & $5(12.5)$ & & \\
\hline Total & $88(91.7)$ & $8(8.3)$ & & \\
\hline
\end{tabular}

${ }^{\mathrm{a}}$ Values are expressed as No. (\%).

cer patients who had been treated with mFOLFOX6 in our study. There was no gastrointestinal toxicity related death (grade 5) as very severe gastrointestinal toxicity in any patients.

There are some risk factors in neurotoxicity induced by oxaliplatin, such as infusion rate, oxaliplatin dose per cycle, total dose, pre-existing peripheral neuropathy, and history of surgery $(18,19)$. The incidence of grade $\geq 1$ neurotoxicity in patients treated with FOLFOX 4 or modified FOLFOX 6 were $10.7 \%$ and 30\% respectively. Our results confirm that the difference between the incidence rate of grade $\geq$ 1 neurotoxicity was significant $(P=0.017)$. Then, FOLFOX 4 scheme was statistically significant better than modified FOLFOX 6 scheme of neurotoxicity.

There are risk factors that have been known concerning oxaliplatin and 5-FU induced hematologic toxicities, including treatment schedule, single dose per cycle, cumulative dose, and infusion time. The difference between the incidence rate of grade $\geq 1$ of neutropenia as hematologic toxicity was very highly significant $(\mathrm{P}<0.001)$, but the difference between grade $\geq 1$ of thrombocytopenia as hematologic toxicity was not significant $(P=0.063)$. Then, FOLFOX 4 scheme was statistically significant higher than modified FOLFOX 6 scheme of neutropenia as hematologic toxicity.

In conclusion, the severity of neutropenia as hematological toxicity, diarrhea and neurotoxicity as nonhematological toxicities were noted in mFOLFOX6. We also showed that in colorectal cancer patients treated with modified FOLFOX6, some of hematological and nonhematological complications were more than FOLFOX4 and they can be concerned.

\section{Acknowledgments}

We would like to thank the technician staff of the radiotherapy department at Shohada-e-Tajrish hospital for their contribution to the treatment and maintenance of our patient records and data collection without whom, carrying out this project would be impossible. 


\section{Footnotes}

Authors' Contribution: None declared.

Conflict of Interests: None declared.

Financial Disclosure: None declared.

\section{References}

1. Siegel R, Ma J, Zou Z, Jemal A. Cancer statistics, 2014. CA Cancer J Clin. 2014;64(1):9-29. doi:10.3322/caac.21208. [PubMed: 24399786].

2. de Gramont A, Figer A, Seymour M, Homerin M, Hmissi A, Cassidy J, et al. Leucovorin and fluorouracil with or without oxaliplatin as first-line treatment in advanced colorectal cancer. J Clin Oncol. 2000;18(16):2938-47. doi: 10.1200/jco.2000.18.16.2938. [PubMed: 10944126].

3. Andre T, Boni C, Mounedji-Boudiaf L, Navarro M, Tabernero J, Hickish $\mathrm{T}$, et al. Oxaliplatin, fluorouracil, and leucovorin as adjuvant treatment for colon cancer. $N$ Engl J Med. 2004;350(23):2343-51. doi: 10.1056/NEJMoa032709. [PubMed: 15175436].

4. Andre T, Boni C, Navarro M, Tabernero J, Hickish T, Topham C, et al. Improved overall survival with oxaliplatin, fluorouracil, and leucovorin as adjuvant treatment in stage II or III colon cancer in the MOSAIC trial. J Clin Oncol. 2009;27(19):3109-16. doi: 10.1200/JCO.2008.20.6771. [PubMed: 19451431].

5. Saltz LB, Clarke S, Diaz-Rubio E, Scheithauer W, Figer A, Wong R, et al. Bevacizumab in combination with oxaliplatin-based chemotherapy as first-line therapy in metastatic colorectal cancer: a randomized phase III study. J Clin Oncol. 2008;26(12):2013-9. doi: 10.1200/JCO.2007.14.9930. [PubMed: 18421054].

6. Bokemeyer C, Bondarenko I, Makhson A, Hartmann JT, Aparicio J, de Braud F, et al. Fluorouracil, leucovorin, and oxaliplatin with and without cetuximab in the first-line treatment of metastatic colorectal cancer. J Clin Oncol. 2009;27(5):663-71. doi: 10.1200/JCO.2008.20.8397. [PubMed: 19114683].

7. Douillard JY, Siena S, Cassidy J, Tabernero J, Burkes R, Barugel $\mathrm{M}$, et al. Randomized, phase III trial of panitumumab with infusional fluorouracil, leucovorin, and oxaliplatin (FOLFOX4) versus FOLFOX4 alone as first-line treatment in patients with previously untreated metastatic colorectal cancer: the PRIME study. J Clin Oncol. 2010;28(31):4697-705. doi: 10.1200/JCO.2009.27.4860. [PubMed: 20921465].

8. Allegra CJ, Yothers G, O'Connell MJ, Sharif S, Colangelo LH, Lopa SH, et al. Initial safety report of NSABP C-08: A randomized phase III study of modified FOLFOX6 with or without bevacizumab for the adjuvant treatment of patients with stage II or III colon cancer. J Clin
Oncol. 2009;27(20):3385-90. doi: 10.1200/JCO.2009.21.9220. [PubMed: 19414665].

9. Carlotto A, Hogsett VL, Maiorini EM, Razulis JG, Sonis ST. The economic burden of toxicities associated with cancer treatment: review of the literature and analysis of nausea and vomiting, diarrhoea, oral mucositis and fatigue. Pharmacoeconomics. 2013;31(9):75366. doi: 10.1007/s40273-013-0081-2. [PubMed: 23963867].

10. Gibson RJ, Keefe DM, Lalla RV, Bateman E, Blijlevens N, Fijlstra M, et al. Systematic review of agents for the management of gastrointestinal mucositis in cancer patients. Support Care Cancer. 2013;21(1):313-26. doi: 10.1007/s00520-012-1644-z. [PubMed: 23142924].

11. Comeau JM, Labruzzo Mohundro B. From bench to bedside: promising colon cancer clinical trials. Am JManag Care. 2013;19(1 Spec No):327. [PubMed: 23461471].

12. Kuebler JP, Wieand HS, O'Connell MJ, Smith RE, Colangelo LH, Yothers $\mathrm{G}$, et al. Oxaliplatin combined with weekly bolus fluorouracil and leucovorin as surgical adjuvant chemotherapy for stage II and III colon cancer: results from NSABP C-07. J Clin Oncol. 2007;25(16):2198-204. doi: 10.1200/JCO.2006.08.2974. [PubMed: 17470851].

13. Hahemi R, Majidi A, Tabatabaey A, Motamed H. Fatal Disseminated Cryptococcus Infection in an Immunocompetent Patient. Arch Clin Infect Dis. 2014;9(3):e20246. doi:10.5812/archcid.20246.

14. Chen Q, Xia HW, Ge XJ, Zhang YC, Tang QL, Bi F. Serum miR-19a predicts resistance to FOLFOX chemotherapy in advanced colorectal cancer cases. Asian Pac J Cancer Prev. 2013;14(12):7421-6. doi: 10.7314/APJCP.2013.14.12.7421. [PubMed: 24460313]

15. Ramanathan RK, Clark JW, Kemeny NE, Lenz HJ, Gococo KO, Haller DG, et al. Safety and toxicity analysis of oxaliplatin combined with fluorouracil or as a single agent in patients with previously treated advanced colorectal cancer. J Clin Oncol. 2003;21(15):2904-11. doi 10.1200/JCO.2003.11.045. [PubMed:12885808].

16. Yousefi Kashi AS, Khaledi S, Houshyari M. CT Simulation to Evaluate of Pelvic Lymph Node Coverage in Conventional Radiotherapy Fields Based on Bone and Vessels Landmarks in Prostate Cancer Patients. Iran J Cancer Prev. 2016;9(3):6233. doi: 10.17795/ijcp-6233. [PubMed: 27703649].

17. Sharif S, O'Connell MJ, Yothers G, Lopa S, Wolmark N. FOLFOX and FLOX regimens for the adjuvant treatment of resected stage II and III colon cancer. Cancer Invest. 2008;26(9):956-63. doi: 10.1080/07357900802132550. [PubMed: 18798075].

18. Uncu D, Aksoy S, Cetin B, Yetisyigit T, Ozdemir N, Berk V, et al. Results of adjuvant FOLFOX regimens in stage III colorectal cancer patients: retrospective analysis of 667 patients. Oncology. 2013;84(4):240-5. doi: 10.1159/000336902. [PubMed: 23392240].

19. Rakhsha A, Yousefi Kashi AS, Hoseini SM. Evaluation of Survival and Treatment Toxicity With High-Dose-Rate Brachytherapy With Cobalt 60 in Carcinoma of Cervix. Iran J Cancer Prev. 2015;8(4):3573. doi: 10.17795/ijcp-3573. [PubMed: 26478798]. 